\title{
Magnetic ordering and negative thermal expansion in PrFeAsO
}

\author{
S. A. J. Kimber, ${ }^{1}$ D. N. Argyriou, ${ }^{1, *}$ F. Yokaichiya, ${ }^{1}$ K. Habicht, ${ }^{1}$ S. Gerischer, ${ }^{1}$ T. Hansen, ${ }^{2}$ T. Chatterji, ${ }^{3}$ R. Klingeler, ${ }^{4}$ \\ C. Hess, ${ }^{4}$ G. Behr, ${ }^{4}$ A. Kondrat, ${ }^{4}$ and B. Büchner ${ }^{4}$ \\ ${ }^{1}$ Helmholtz-Zentrum Berlin für Materialien und Energie (HZB), Glienicker Strasse 100, D-14109 Berlin, Germany \\ ${ }^{2}$ Institute Max von Laue-Paul Langevin, 6 rue Jules Horowitz, BP 156, F-38042 Grenoble Cedex 9, France \\ ${ }^{3}$ JCNS, Forschungszentrum Jülich Outstation at Institut Laue-Langevin, BP 156, F-38042 Grenoble Cedex 9, France \\ ${ }^{4}$ Leibniz-Institute for Solid State and Materials Research (IFW), Dresden, Germany \\ (Received 26 July 2008; revised manuscript received 4 September 2008; published 2 October 2008)
}

\begin{abstract}
We report the structure and magnetism of $\mathrm{PrFeAsO}$, one of the parent phases of the Fe-As family of superconductors, as measured by neutron powder diffraction. In common with other REFeAsO materials, a tetragonal-orthorhombic phase transition is found on cooling below $136 \mathrm{~K}$ and striped Fe magnetism with $k$ $=(1,0,1)$ is detected below $\sim 85 \mathrm{~K}$. Our magnetic order-parameter measurements show that the ordered Fe moment along the $a$ axis reaches a maximum at $\sim 40 \mathrm{~K}$, below which an anomalous expansion of the $c$ axis sets in that results in a negative thermal volume expansion of $0.015 \%$ at $2 \mathrm{~K}$. We propose that this effect, which is suppressed in superconducting samples, is driven by a delicate interplay between $\mathrm{Fe}$ and $\mathrm{Pr}$ ordered moments.
\end{abstract}

DOI: 10.1103/PhysRevB.78.140503

PACS number(s): 74.25.Ha

Until recently, and despite decades of research, no high $T_{c}$ superconductors except the cuprate family had been foundperhaps due to the perceived wisdom that the only likely candidates were doped Mott insulators. The discovery of superconductivity at $25 \mathrm{~K}$ in $\mathrm{LaFeAsO}_{1-x} \mathrm{~F}_{x}$ (Ref. 1) has therefore caused much interest. Like the cuprates, the REFeAsO ( $\mathrm{RE}=$ rare earth) family of compounds is layered; however, the parent phases are bad metals and iron is in a tetrahedral coordination with arsenic, in contrast to the square planar geometry of the cuprates. On cooling, the undoped parent phases undergo an electronic transition at $T_{s} \sim 150 \mathrm{~K}$ at which the resistivity and magnetization drop sharply. Initial powder-diffraction studies showed that this transition corresponds to a structural change from tetragonal to orthorhombic symmetry, ${ }^{2}$ and at slightly lower temperatures, commensurate Fe-spin order develops. ${ }^{3,4}$

Initially, many authors speculated that the structural distortion is the result of a spin-density wave transition and that superconductivity arises as a result of doping, which suppresses both the structural phase transition and the Fe-spin ordering. ${ }^{5}$ On this basis, strong analogies have been drawn with the cuprate materials. Alternatively, as the Fe-As layers in the high-temperature tetragonal phase of these compounds are a perfect realization of the $J_{1}-J_{2}$ frustrated square lattice, magnetic frustration has also been proposed as a potential driving force for the symmetry lowering phase transition at $150 \mathrm{~K} .{ }^{6}$ Indeed, the magnetic structures reported for the majority of the undoped compounds are simple collinear striped models, which are predicted to be the ground state of the $J_{1}-J_{2}$ model when $J_{1} / J_{2} \sim 1$. Evidence for coupling between structural and magnetic order parameters is particularly strong in the $A \mathrm{Fe}_{2} \mathrm{As}_{2}(A=\mathrm{Ca}, \mathrm{Sr}, \mathrm{Ba})$ family of materials. Neutron-diffraction studies show that magnetic order emerges exactly at the structural transition. ${ }^{7-9}$ In the REFeAsO compounds, however, the structural and magnetic transitions are well separated; in LaFeAsO (Ref. 2) and $\mathrm{CeFeAsO},{ }^{4}$ spin order is found some $20 \mathrm{~K}$ below $T_{s}$, suggesting a weaker coupling.

In this Rapid Communications, we describe a series of neutron-scattering experiments, which show that in $\mathrm{PrFeAsO}$ there is a magnetoelastic coupling that results in a negative thermal expansion (NTE) of the $c$ axis below $\sim 40 \mathrm{~K}$ and an expansion of the unit-cell volume. While for this compound $T_{s}=136 \mathrm{~K}$, our measurements become sensitive to Fe magnetic order much below $T_{s}$ at a tentative $T_{N}(\mathrm{Fe})=85 \mathrm{~K}$, in which the Fe spins order in the form of antiferromagnetically coupled stripes with the moments pointing along the $a$ axis and propagation vector $k=(1,0,1)$. The Pr spins are found to order below $T_{N}(\operatorname{Pr})=12 \mathrm{~K}$. The emergence of the lattice anomaly correlates with changes in the intensity of the (1 $\overline{1} 2)$ magnetic reflection, and is likely driven by a subtle interplay between Pr- and Fe-spin orders. Our diffraction measurements show that the NTE behavior is suppressed in superconducting $\mathrm{PrFeAsO}_{0.85} \mathrm{~F}_{0.15}$ together with magnetic order.

We synthesized polycrystalline samples of $\mathrm{PrFeAsO}$ and $\mathrm{PrFeAsO}_{0.85} \mathrm{~F}_{0.15}$ by the previously reported methods. ${ }^{10}$ The resistivity data for PrFeAsO shown in Fig. 1 exhibit several anomalies below $300 \mathrm{~K}$. The most prominent feature is a broad maximum around $T_{s}$. While the resistivity increases when approaching $T_{s}$ from higher temperatures, there is a pronounced suppression of $\rho$ around $150 \mathrm{~K}$. Such a behavior is typical for the REFeAsO materials. Interestingly, similar to, e.g., LaFeAsO, there is a kink in the derivative $d \rho / d T$ at a temperature slightly below $T_{s} \cdot{ }^{11}$ From the comparison with $\mathrm{LaFeAsO}$ we conclude that this kink indicates the onset of long-range spin order as indirectly probed by the electrical resistivity, i.e., $T_{N}^{\rho}(\mathrm{Fe}) \sim 127 \mathrm{~K}$. Note that in contrast to $\mathrm{LaFeAsO}$, large $\mathrm{RE}$ moments are present in $\mathrm{PrFeAsO}$, which mask any anomaly in our static magnetization data around $T_{s}$. An additional anomaly, i.e., a sharp decrease in the resistivity, is observed at much lower temperatures, i.e., $T_{N}(\mathrm{Pr})$ $=11 \mathrm{~K}$. Our neutron data (see below) confirm that, at this temperature, magnetic order of the Pr spins evolves. Our fluorine doped sample shows a superconducting transition with a $T_{C}=43 \mathrm{~K}$. Neutron powder-diffraction (NPD) measurements were measured as a function of temperature on the D20 powder diffractometer, ${ }^{12}$ located at the Institut Laue- 


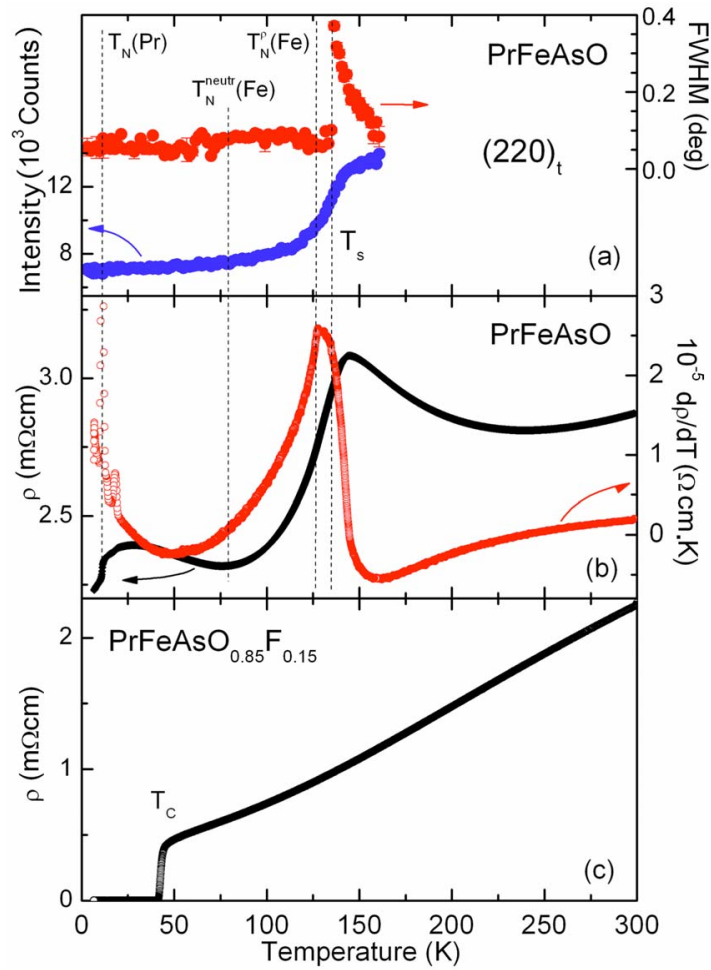

FIG. 1. (Color online) (a) Temperature evolution of the PrOFeAs $(220)_{t}$ peak, as measured at $2 \theta=82.3^{\circ}$, and FWHM of the $(220)_{t}$ and $(400)_{o} /(040)_{o}$ peaks. (b) Resistivity measurements from the $\mathrm{PrFeAsO}$ and (c) superconducting $\mathrm{PrFeAsO}_{0.85} \mathrm{~F}_{0.15}$ samples.

Langevin, using a wavelength of $\lambda=1.88 \AA$. Additional NPD data were also measured using the E9 high-resolution neutron powder diffractometer with $\lambda=1.79 \AA$ and the FLEX cold triple axis spectrometer with $\lambda=4.05 \AA$, both at
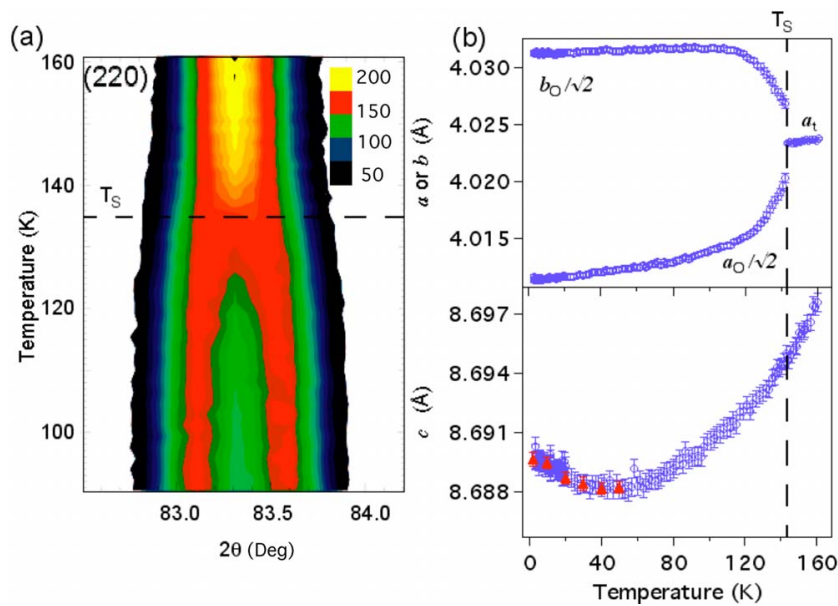

FIG. 2. (Color online) (a) Temperature evolution of the (220) peak in PrFeAsO (D20 data) showing the orthorhombic splitting at $T_{s}$. The inset shows intensity scale in units of counts $\times 10^{3}$. (b) Temperature dependence of the $a$ and $b$ axes (top), and $c$ axis (bottom) determined from Rietveld analysis of the neutron powderdiffraction data. A clear anomalous expansion of the $c$ axis, which corresponds to the layer stacking direction, is observed below $50 \mathrm{~K}$. This expansion is confirmed by measurement on the E9 diffractometer shown on the same panel as (blue) open symbols.

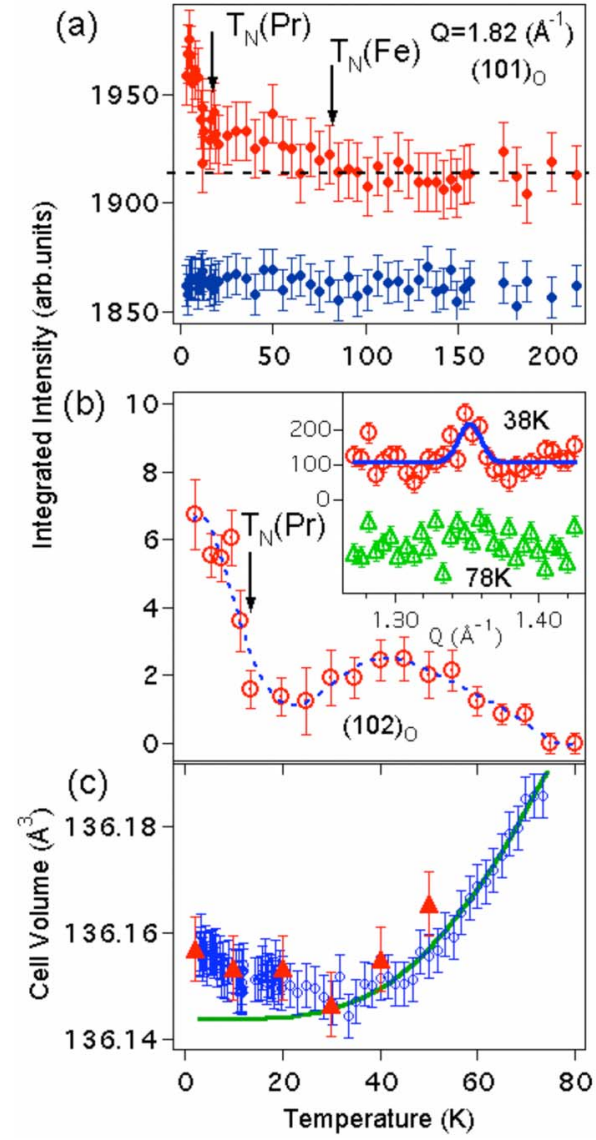

FIG. 3. (Color online) (a) Magnetic order parameter for PrOFeAs measured at the (102)o reflection; (b) magnetic order parameter measured at the (101) reflection; (c) refined cell volume of $\mathrm{PrFe} A s \mathrm{O}$ from neutron powder-diffraction data, in which red circles represent data collected using D20 at the ILL and blue triangles are data collected using E9 at HZB. Also shown is a Debye fit to the high-temperature $(50-120 \mathrm{~K})$ cell volume.

HZB. The NPD data were analyzed using the Rietveld method with the programs GSAS (Ref. 13) and FULLPROF. ${ }^{14}$ We independently refined all site occupancies, all of which are refined to within error of unity. Together with the absence of impurity phases, this indicates that our sample is stoichiometric to within an estimated error of $1 \%$. Turning our attention on the NPD measurements from D20, we find that on cooling the PrFeAsO sample the structural phase transition from $P 4 / \mathrm{nmm}$ to $C m m a$ occurs at $T_{s}=136 \mathrm{~K}$, similar to what has been reported for the other REFeAsO materials. ${ }^{2-4}$ The evolution with temperature of the $(220)$, tetragonal reflection, which splits on cooling into the $(400)_{o}$ and $(040)_{o}$ reflections, is shown in Fig. 2(a). The sharp correlation between the resistivity decrease at $136 \mathrm{~K}$ and the structural phase transition is highlighted by plotting the intensity of the $(220)_{t}$ peak from the NPD data between $83.3^{\circ}$ and $83.4^{\circ}$ as a function of temperature (Fig. 1). The exact determination of the transition temperature from the NPD data is challenging as the nature of this transition appears to be first order. ${ }^{2,9}$ The exact transition temperature cannot be established simply by the sequential Rietveld refinements of the NPD data as the data can be almost equally well fitted just above or below $T_{s}$ 

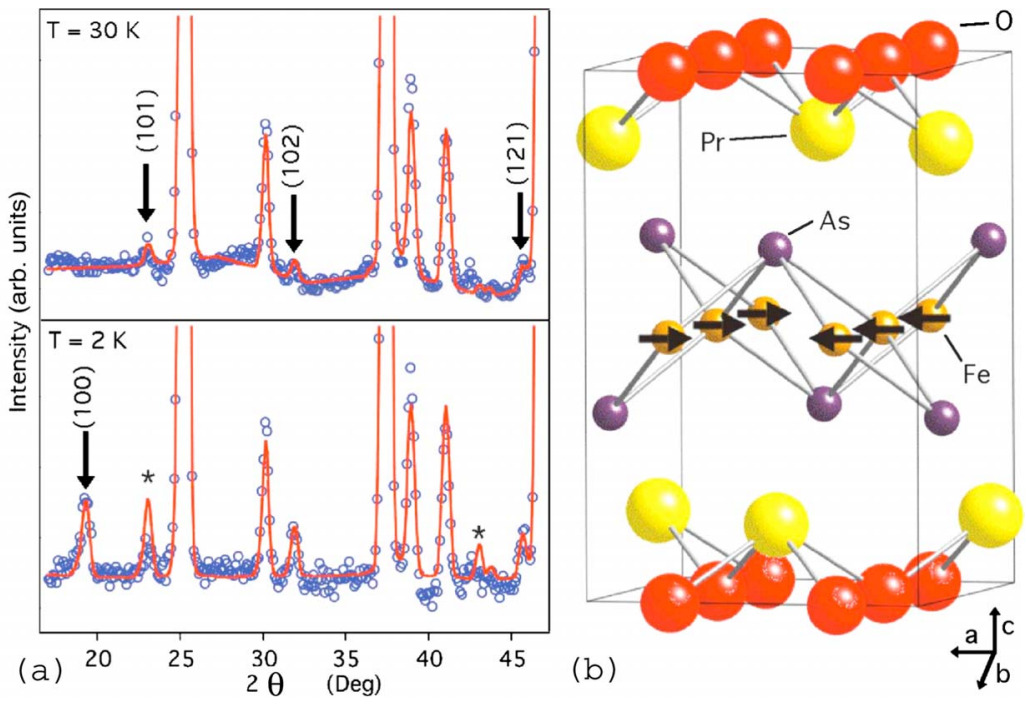

FIG. 4. (Color online) (a) Low angle region of neutron powder-diffraction patterns for $\mathrm{PrFeAsO}$, recorded using D20. Top pattern shows fit at $30 \mathrm{~K}$ in Fe-spin ordered regime while bottom pattern shows combined $\mathrm{Fe}$ and $\mathrm{Pr}$ fit at $1.4 \mathrm{~K}$. Stars mark areas of disagreement between observed and calculated patterns; (b) Refined Fe magnetic structure of $\mathrm{PrFeAsO}$ at $30 \mathrm{~K}$. with either the Cmma or $P 4 / \mathrm{nmm}$ structures. A better method to analyze the splitting of the $(220)_{t}$ reflection close to $T_{s}$ is to model it with either one or two resolution limited reflections. This defines the transition temperature (for example on cooling) as the point of which the $(220)_{t}$ reflection becomes significantly broader than the resolution of the diffractometer to support modeling by two peaks. Such analysis shows that the full width at half maximum (FWHM) of the $(400)_{o} /(040)_{o}$ reflection sharply increases at $T_{s}=136 \mathrm{~K}$, in excellent agreement with that obtained from the resistivity measurements, while the $(220)_{t}$ reflection is significantly broader above $T_{s}$. A physical interpretation of such analysis would suggest an abrupt transition on warming, with an extended range of coexistance, as a broader peak within the resolution of the measurement. The effect of the structural distortion on the cell parameters is also shown in Fig. 2(b). The $a$ axis is compressed and the $b$ axis expands, leading to inequivalent $J_{[100]}$ and $J_{[010]}$ interactions in the Fe-As planes as the Fe-Fe distances and Fe-As-Fe bond angles change. These structural changes lift the magnetic frustration, allowing the development of Fe-spin order (see below) at lower temperature. Surprisingly on further cooling below $\sim 45 \mathrm{~K}$, the $c$ axis (which corresponds to the layer stacking direction) smoothly expands. As shown in Fig. 3, this effect dominates the unit-cell volume at low temperature, leading to a small but measurable negative thermal expansion of the volume (see below). This increase in the $c$ axis was confirmed at low temperatures, also on the E9 diffractometer at HZB, in a constant-temperature mode of operation [shown as black circles in Fig. 2(c)], confirming our measurements on D20. In order to estimate the size of the effect, we fitted the hightemperature $(50-160 \mathrm{~K})$ cell volume to a second-order Grüneisen approximation yielding a Debye temperature $\theta_{D}$ $=355(5) \mathrm{K} .{ }^{15}$ The extrapolated cell volume at low temperature is shown as a solid line in Fig. 3. Comparing the expected unit-cell volume on the basis of the second-order Grüneisen fitting, we calculate a volume change of approximately $0.015 \%$ at $1.4 \mathrm{~K}$, which is remarkably similar in magnitude to the magnetoelastic volume effect and negative thermal expansion reported in other frustrated magnets, such as the spinels $\mathrm{ZnCr}_{2} \mathrm{Ch}_{4}(\mathrm{Ch}=\mathrm{S}, \mathrm{Se}) .{ }^{16}$ We propose that negative thermal expansion in $\mathrm{PrFe} A s \mathrm{O}$ originates in magnetoelastic coupling related to the onset of Fe- and Pr-spin orders. Below $T_{S}$ we find evidence of magnetic ordering arising from $\mathrm{Pr}$ and $\mathrm{Fe}$ spins. On cooling below $T_{s}$ we observe the emergence of magnetic reflections, the most prominent being the (101) and (102). These reflections index on the chemical cell and correspond to a simple striped model of $\mathrm{Fe}$ spins with moments of $0.35(5) \mu_{B}$ along the $a$ axis with $k$ $=(1,0,1)$ at $30 \mathrm{~K}$, as shown in Fig. 4 . Note that this wave vector is equivalent to $k=(0,0,0)$ in a primitive cell. At $12 \mathrm{~K}$ we also find that Pr spins order, giving a sharp rise in the intensity of these magnetic reflections as well as the observation of a (100) magnetic reflection. The best fit to the low-temperature NPD data was achieved by a model where both Fe and Pr spins order within the $a b$ plane in the twoleft, one-right magnetic structure described for $\mathrm{NdFeAsO}{ }^{3}$ At $1.4 \mathrm{~K}$ the moments of the $\mathrm{Fe}$ and Pr ions are $0.53(20) \mu_{B}$ and $0.83(9) \mu_{B}$, respectively. We searched extensively but unsuccessfully for reflections that would justify a change in the magnetic propagation vector below $12 \mathrm{~K}$, as was reported for $\mathrm{RE}=\mathrm{Ce} .^{4}$ In addition we attempted to fit the magnetic scattering at low temperature with the Fe-spin model described above, and various arrangements of Pr moments with and without doubling of the $c$ axis. The model described above provided the best fit to the data by far. The onset of the Prand Fe-spin orderings was determined by following the temperature dependence of the magnetic reflections using both the D20 data as well as performing additional measurements on the FLEX spectrometer. In both measurements, the magnetic scattering is extremely weak but is commensurate with the lattice at all temperatures, confirming its origin. The temperature dependence of the (101) and (102) magnetic reflections are shown in Figs. 4(a) and 4(b). Given the small size of the ordered Fe moment, the D20 data were binned in approximately $5 \mathrm{~K}$ intervals to enhance the statistics of a given diffraction pattern. To obtain the temperature dependence of the (102) reflection, the data were integrated over a $1.5^{\circ}$ range around the peak. A similar size range of $2 \theta$ adjacent to the (102) reflection was also integrated to gain an estimate of the background and its temperature dependence, which was found to be constant. The results of this analysis 
are plotted in Fig. 3(a). We find that, although there is a well defined Bragg reflection at low temperature, the magnetic scattering becomes much weaker as temperature is increased toward $T_{s}$. A sharp drop in the intensity of this reflection is observed on warming through $12 \mathrm{~K}$, which signifies the onset of Pr ordering, and the magnetic scattering finally disappears above $85 \mathrm{~K}$. This observation will tentatively place $T_{N}(\mathrm{Fe}) \sim 85 \mathrm{~K}$; however, in the present investigation we are only sensitive to magnetic scattering once the Fe moment reaches a value of $0.1 \mu_{B}$. More sensitive probes to Fe magnetism such as muon and Mössbauer spectroscopy may well place $T_{N}(\mathrm{Fe})$ to be either coincident with $T_{s}$ or a few Kelvin below, as has been found for $\mathrm{RE}=\mathrm{La}, \mathrm{Ce}$, and $\mathrm{Nd} .^{2-4}$

We followed the temperature dependence of the (101) reflection with the FLEX triple axis spectrometer and found its evolution to be somewhat different from that of the (102). A large decrease is also observed for this reflection on warming through $T_{N}(\operatorname{Pr})$; however, the scattering from the Fe-spin order reaches a plateau around $40 \mathrm{~K}$ before disappearing above $75 \mathrm{~K}$. The apparent lower $T_{N}(\mathrm{Fe})$ given by this reflection likely arises from its smaller magnetic structure factor compared to the (102). As is evident in Fig. 3 the expansion of the unit-cell volume coincides with the plateau of the (101) reflection, suggesting that its origin is magnetoelastic. The rise in intensity of the (101) Fe magnetic reflection up to $\sim 40 \mathrm{~K}$ and its decrease as $T_{N}(\operatorname{Pr})$ is approached, suggests a possible interplay between $\mathrm{Pr}$ and Fe. One possible explanation is a decrease in the ordered Fe moment along the $a$ axis due to the influence of the Pr moments. Such behavior has also been alluded to in Ref. 4 for $\mathrm{CeFeAsO}$; however, in the present case the magnetoelastic coupling generated is sufficiently strong to produce an increase in the unit-cell volume.
Such effects have not been observed to date in the REFeAsO family of compounds.

We also performed neutron powder-diffraction measurements on superconducting $\mathrm{PrFeAsO}_{0.85} \mathrm{~F}_{0.15}$ down to $0.4 \mathrm{~K}$ using a ${ }^{3} \mathrm{He}$ insert. We detect no evidence for Fe or Pr magnetic order while the NPD data show a positive thermal expansion for the $a$ and the $c$ axes. This result is also in contrast to measurements for cuprate superconductors such as $\mathrm{Nd}_{2-x} \mathrm{Ce}_{x} \mathrm{CuO}_{4}$, in which rare-earth antiferromagnetism is found to coexist with the superconducting state ${ }^{17}$ below 2.5 $\mathrm{K}$. This would indicate that the rare-earth magnetism in the layered FeAs compounds may be induced by the Fe-spin order.

In conclusion, our measurements show that $\mathrm{PrFe} A s \mathrm{O}$ undergoes the same $T-O$ phase transition as the other REFeAsO compounds at $136 \mathrm{~K}$. On cooling below $\sim 85 \mathrm{~K}$ we detect long-range magnetic order of the $\mathrm{Fe}$ moments in a striped arrangement with $k=(1,0,1)$ and at lower temperatures $(12$ K) long-range order of Pr spins. Our detailed Rietveld refinements have identified a previously unknown coupling of magnetic and lattice degrees of freedom at $\sim 40 \mathrm{~K}$, which leads to an anomalous expansion of the layer stacking direction. We propose that this negative thermal-expansion results from a delicate interplay between the ordered Fe and Pr sublattices. Suppression of the NTE state and long-range magnetic order is necessary for the emergence of superconductivity in $\operatorname{Pr}_{1-x} \mathrm{~F}_{x} \mathrm{FeAsO}$.

Note Added. Recently, Zhao et al. ${ }^{18}$ presented data which are in agreement with our results.

We acknowledge the Helmholtz Zentrum Berlin for funding and the Institute Max von Laue-Paul Langevin for access to their instruments.
*Corresponding author. argyriou@helmholtz-berlin.de

${ }^{1}$ Y. Kamihara, T. Watanabe, M. Hirano, and H. Hosono, J. Am. Chem. Soc. 130, 3296 (2008).

${ }^{2}$ C. de la Cruz et al., Nature (London) 453, 899 (2008).

${ }^{3}$ Y. Chen, J. W. Lynn, J. Li, G. Li, G. F. Chen, J. L. Luo, N. L. Wang, P. Dai, C. dela Cruz, and H. A. Mook, Phys. Rev. B 78, 064515 (2008).

${ }^{4}$ J. Zhao, Q. Huang, C. de la Cruz, S. Li, J. W. Lynn, Y. Chen, M. A. Green, G. F. Chen, G. Li, Z. Li, J. L. Luo, N. L. Wang, and P. Dai, arXiv:0806.2528 (unpublished).

${ }^{5}$ J. Dong, H. J. Zhang, W. Z. Hu, G. F. Chen, X. Dai, J. L. Luo, Z. Fang, and N. L. Wang, Europhys. Lett. 83, 27006 (2008).

${ }^{6}$ T. Yildirim, Phys. Rev. Lett. 101, 057010 (2008).

${ }^{7}$ Q. Huang, Y. Qiu, W. Bao, J. W. Lynn, M. A. Green, Y. Chen, T. Wu, G. Wu, and X. H. Chen, arXiv:0806.2776 (unpublished).

${ }^{8}$ J. Zhao, W. Ratcliff II, J. W. Lynn, G. F. Chen, J. L. Luo, N. L. Wang, J. Hu, and P. Dai, arXiv:0807.1077 (unpublished).

${ }^{9}$ A. I. Goldman, D. N. Argyriou, B. Ouladdiaf, T. Chatterji, A. Kreyssig, S. Nandi, N. Ni, S. L. Bud'ko, P. C. Canfield, and R. J. McQueeney, Phys. Rev. B 78, 100506(R) (2008).

${ }^{10}$ X. Zhu, H. Yang, L. Fang, G. Mu, and H.-H. Wen, Supercond.
Sci. Technol. 21, 105001 (2008).

${ }^{11}$ H. H. Klauss, H. Luetkens, R. Klingeler, C. Hess, F. J. Litterst, M. Kraken, M. M. Korshunov, I. Eremin, S. L. Drechsler, R. Khasanov, A. Amato, J. Hamann-Borrero, N. Leps, A. Kondrat, G. Behr, J. Werner, and B. Buchner, Phys. Rev. Lett. 101, 077005 (2008).

${ }^{12}$ T. Hansen, P. F. Henry, H. E. Fischer, J. Torregrossa, P. Convert, and H. A. Convert, Meas. Sci. Technol. 19, 034001 (2008).

${ }^{13}$ A. Larson and R. V. Dreele, Los Alamos National Laboratory Report No. LAUR 86-748, 1998 (unpublished).

${ }^{14}$ J. Rodriguez-Carvajal, Physica B 192, 55 (1993).

${ }^{15}$ L. Vočadlo, K. Knight, G. Price, and I. Wood, Phys. Chem. Miner. 29, 7169 (2002).

${ }^{16}$ J. Hemberger, H. A. Krug von Nidda, V. Tsurkan, and A. Loidl, Phys. Rev. Lett. 98, 147203 (2007).

${ }^{17}$ J. W. Lynn, I. W. Sumarlin, S. Skanthakumar, W. H. Li, R. N. Shelton, J. L. Peng, Z. Fisk, and S. W. Cheong, Phys. Rev. B 41, 2569 (1990).

${ }^{18}$ J. Zhao, Q. Huang, C. de la Cruz, J. W. Lynn, M. D. Lumsden, Z. A. Ren, J. Yang, X. Shen, X. Dong, Z. Zhao, and P. Dai, arXiv:0807.4872 (unpublished). 